\title{
Time Period From Onset of Pain to Hospital Admission and Patients' Awareness in Acute Pancreatitis
}

\author{
Veit Phillip, MD, * Tibor Schuster, PhD, † Frank Hagemes, * Sandra Lorenz, * Ulrike Matheis, * \\ Sigrid Preinfalk, * Florian Lippl, MD, $\neq$ Bernd Saugel, MD, * Roland M. Schmid, MD, * \\ and Wolfgang Huber, $M D^{*}$
}

Objectives: This study aimed to explore the period between onset of
pain and hospital-admission (pain-to-admission time) in patients with
acute pancreatitis (AP), to investigate the prognostic value and associ-
ated factors of this time, and to ascertain the knowledge about the pan-
creas in these patients.

Methods: An analysis of a prospective multicenter study was done, which included 188 patients with AP.

Results: Median pain-to-admission time was 27 hours (interquartile range, 6.0-72.0). Median pain-to-admission time was significantly shorter in intensive care unit (ICU) patients (10 hours) compared to non-ICU patients (36 hours) $(P=0.045)$. Short pain-to-admission time was associated with high pain level. Median pain level ( 0 , no pain; 10 , maximal pain) was 8.0 (interquartile range, 7.0-10.0). Older age correlated with lower pain level $(r=-0.26 ; P=0.002)$. Multiple logistic regression analysis including the admission values for serum lipase and C-reactive protein and the corresponding interactions to the painto-admission time showed substantial discriminative ability regarding ICU admission (concordance index, 0.706; $P=0.006) .86 \%(112 / 130)$ knew that they have a pancreas, $72 \%(81 / 112)$ of these patients knew that AP exists, and $56 \%(45 / 81)$ recognized that AP is potentially fatal. Conclusions: Knowledge about AP in hospitalized AP patients is poor. Serum lipase and C-reactive protein in dependency of the painto-admission time might be a suitable predictor for severity of AP.

Key Words: prognostic value, C-reactive protein, serum lipase, time to admission

(Pancreas 2013;42: 647-654)

A cute pancreatitis (AP) is a severe and potential lifethreatening disease with an incidence of 19.7 to 42.8 / $100,000 / y^{1,2}$ Although approximately $85 \%$ of AP episodes are mild and self-limiting, severe cases have a mortality of up to $42 \%{ }^{3,4}$ The diagnosis is based on typical clinical symptoms and laboratory tests. ${ }^{5}$ Early diagnosis and immediate therapyelimination of the causing agent, intravenous fluid replacement, and analgesics - are of high importance in the management

From the *II. Medizinische Klinik und Poliklinik, †Institut für Medizinische Statistik und Epidemiologie, Klinikum rechts der Isar der Technischen Universität München; and \$Medizinische Klinik, Ludwig-MaximilianUniversität, Campus Innenstadt, München, Germany.

Received for publication January 5, 2012; accepted August 27, 2012.

Reprints: Veit Phillip, MD, II. Medizinische Klinik und Poliklinik, Klinikum rechts der Isar der Technischen Universität München, Ismaninger Straße 22, 81675 München, Germany (e-mail: veit.phillip@lrz.tum.de).

This study was supported by the Deutsche Forschungsgemeinschaft (HU1707-2-1) (Dr Huber).

The authors declare no conflict of interest.

Supplemental digital contents are available for this article. Direct URL citations appear in the printed text and are provided in the HTML and PDF versions of this article on the journal's Web site (www.pancreasjournal.com).

Copyright $(2013$ by Lippincott Williams \& Wilkins of AP. ${ }^{5-9}$ To be able to start therapy timely, the patient has to recognize AP-induced pain, to appropriately estimate pain severity, and to show up in the emergency department as soon as possible. The pain-to-admission time might be shortened if the patient has knowledge of the existence of the pancreas, where the pancreas is located, that the pancreas can get inflamed, can cause pain, and that this is a good reason to consult a physician.

The aim of this extended analysis of a previously published prospective study on AP was to determine the period between onset of pain and admission to the hospital (pain-to-admission time) in patients experiencing AP and to determine the influencing factors associated with the delay as well as the prognostic value of this period. A further aim was to investigate the knowledge about the pancreas and AP in hospitalized patients experiencing AP.

\section{MATERIALS AND METHODS}

This is an extended analysis of data from a prospective multicenter study by our group on the incidence of AP. ${ }^{2}$ Evaluation of the patients' first symptoms, knowledge on AP, initial management, and pain-to-admission time were a priori end points of the prospective multicenter PROST (Pancreatitis during Oktoberfest) study. This was a prospective study of 188 patients with AP, treated at 27 hospitals in the greater area of Munich, Germany (2,970,000 inhabitants) during the 2008 Oktoberfest and showed that the incidence of AP does not rise during the world's largest beer fair compared with two 18-day control periods but is higher than previously described in Germany. All patients admitted to the hospital because of AP were interviewed using a questionnaire. They were asked about the onset of pain, about their own explanation of the symptoms, and their personal measures against their complaints. The painto-admission time was rounded to the nearest hour. Additionally, patients were explored if they ever had heard about AP, and if they thought AP was a dangerous disease. Pain level was measured on a scale from 0 (no pain) to 10 (maximal pain).

\section{Statistical Analysis}

Statistical analyses were performed using IBM SPSS Statistics software (version 19, SPSS Inc, Chicago, Ill). Descriptive data are presented as median and interquartile range (IQR). In case of missing values, proportions were calculated based on the number of valid data. To compare quantitative data between 2 independent subgroups, Mann-Whitney $U$ test was performed. Spearman correlation coefficient $(r)$ was calculated to assess bivariate relationship of ordinal or continuous parameters. Parameters with a $P<0.1$ in univariable analysis and a limited number of factors with high probability of impact were considered for multivariable logistic regression analyses regarding dichotomous end points. To assess the discriminative ability of the resulting regression models, the concordance index (c-index $=$ area under the curve) was calculated based on the resulting predicted probabilities by a receiver operating characteristics 
TABLE 1. Patients Characteristics $(n=188)$

\section{Age, y}

Sex, male

Body height, $\mathrm{cm}$

Body weight, $\mathrm{kg}$

Body mass index, $\mathrm{kg} / \mathrm{m}^{2}$

Residence, in town

Biliary AP

Alcoholic AP

Time between onset of pain and hospital admission, $\mathrm{h}$

Pain level, 1-10

Where applicable, data are presented as median with IQR $(25$ th -75 th percentile); otherwise, number (\%).

analysis. Piecewise log-linear models were used to assess relative impact of ordinal- or quantitative data on right-skewed outcome variables as pain-to-admission time. All statistical tests performed were 2 -sided and a $P<0.05$ was considered to indicate statistical significance.

\section{RESULTS}

\section{Patients' Characteristics}

Patients' characteristics are shown in Table 1. Of the 188 patients, $128(68 \%)$ answered all questions about their knowledge on the pancreas and AP.

\section{Pain Level}

In general, patients experienced severe pain. The median pain level on a scale from 0 to 10 was 8.0 (IQR, 7.0-10.0) on hospital admission. Distribution of the pain levels is shown in Figure 1. There was a weak but statistically significant inverse correlation between age and pain level $(r=-0.26 ; P=0.002)$.

\section{Pain-to-Admission Time}

The median pain-to-admission time was 27 hours (range, 0-672; IQR, 6.0-72.0 hours). Despite marked pain, only 50\% of patients were admitted to the hospital within 24 hours after

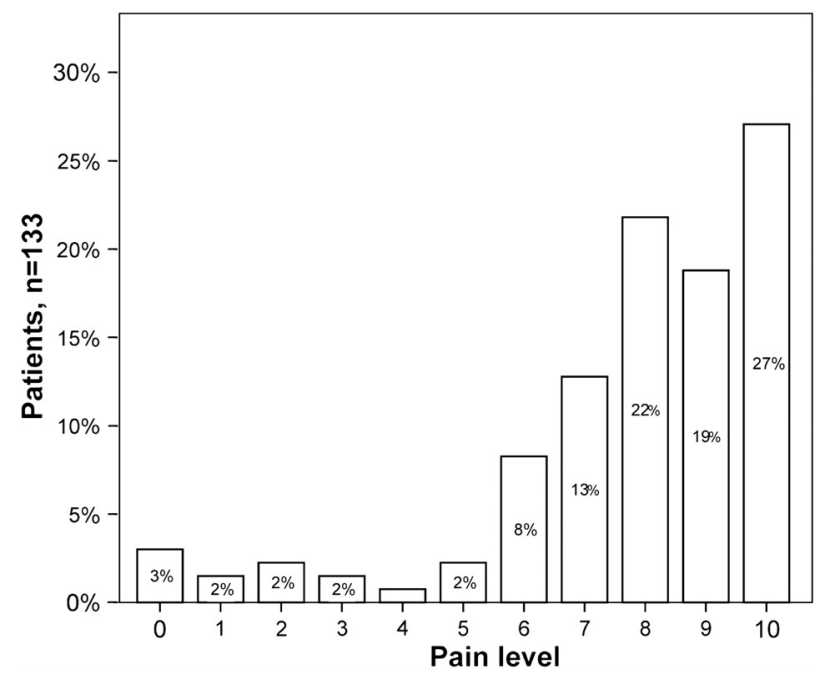

FIGURE 1. Bar plot showing the distribution of the pain levels 0 (no pain) to 10 (maximal pain).

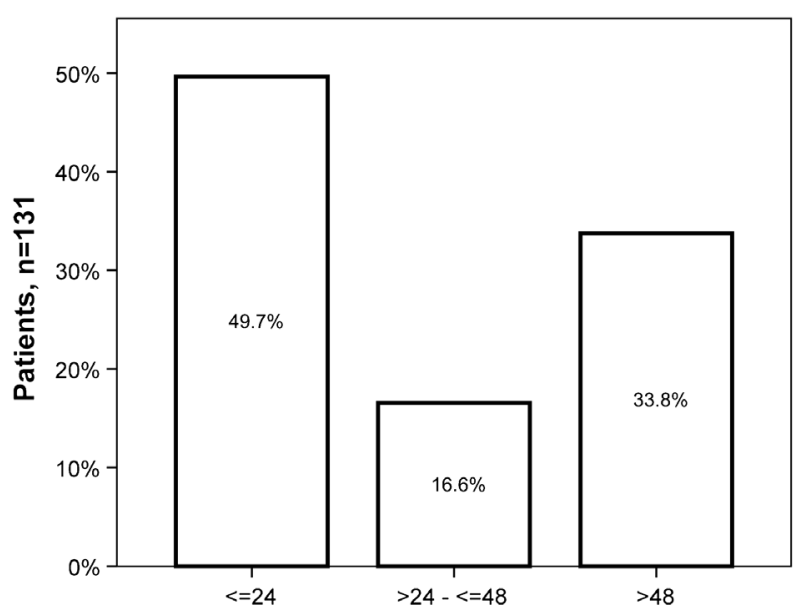

Time between onset of pain and hospital admission [hours]

FIGURE 2. Bar plot showing the distribution of patients admitted to the hospital within categorized time intervals.

onset of pain (Fig. 2). For pain levels greater or equal than 6 , the pain-to-admission time significantly decreased with increasing pain level [decrease for each 1-U pain level, 27\%; 95\% confidence interval (CI), 59\%-89\%; $P=0.003$ ] (see Fig. 1, Supplemental Digital Content 1, http://links.lww.com/MPA/A203).

Univariable comparison of the pain-to-admission time revealed no significant differences between the categories of the parameters sex $(P=0.407)$, etiology (alcoholic, $P=0.262$; biliary, $P=0.593$ ), principal residence (city of Munich or surrounding area, $P=0.618)$, and the knowledge that AP is a potential fatal disease $(P=0.250)$.

\section{Prognostic Factors on Hospital Presentation \\ C-Reactive Protein}

Low values of C-reactive protein (CRP) on admission were associated with short pain-to-admission time $(r=+0.334 ; P<$ $0.001)$. Furthermore, low values of CRP on admission weakly correlated with high pain intensity $(r=-0.208 ; P=0.021)$. The median time from onset of pain to maximum value of CRP was 3 days (IQR, 2-5 days; Fig. 3A). The highest maximum values of CRP were also observed around day 3 after onset of pain (Fig. 3B). The median of maximum CRP values was $13.9 \mathrm{mg} / \mathrm{dL}$ (IQR, 3.3-26.2 mg/dL).

\section{Lipase}

Whereas patients with early presentation had lower CRP levels (see previously mentioned), short pain-to-admission time correlated with higher serum values of lipase on admission $(r=-0.226 ; P=0.019)$. In $38 / 110(35 \%)$ patients, the highest value of serum lipase was measured within the first day after onset of pain (Fig. 4A). The median time from onset of pain to highest maximum values of lipase was 2 days (IQR, 0.4-3.8 days; Fig. 4B) with a median of maximum lipase values of $1211 \mathrm{U} / \mathrm{L}$ (IQR 433-2668 U/L). Serum lipase values on admission did not considerably correlate with the pain level $(r=+0.176 ; P=0.083)$.

\section{Prognostic Value of the Pain-to-Admission Time in Association With CRP and Serum Lipase}

The pain-to-admission time was significantly shorter in intensive care unit (ICU) patients (10 hours) compared to nonICU patients (36 hours; $P=0.045$ ). 

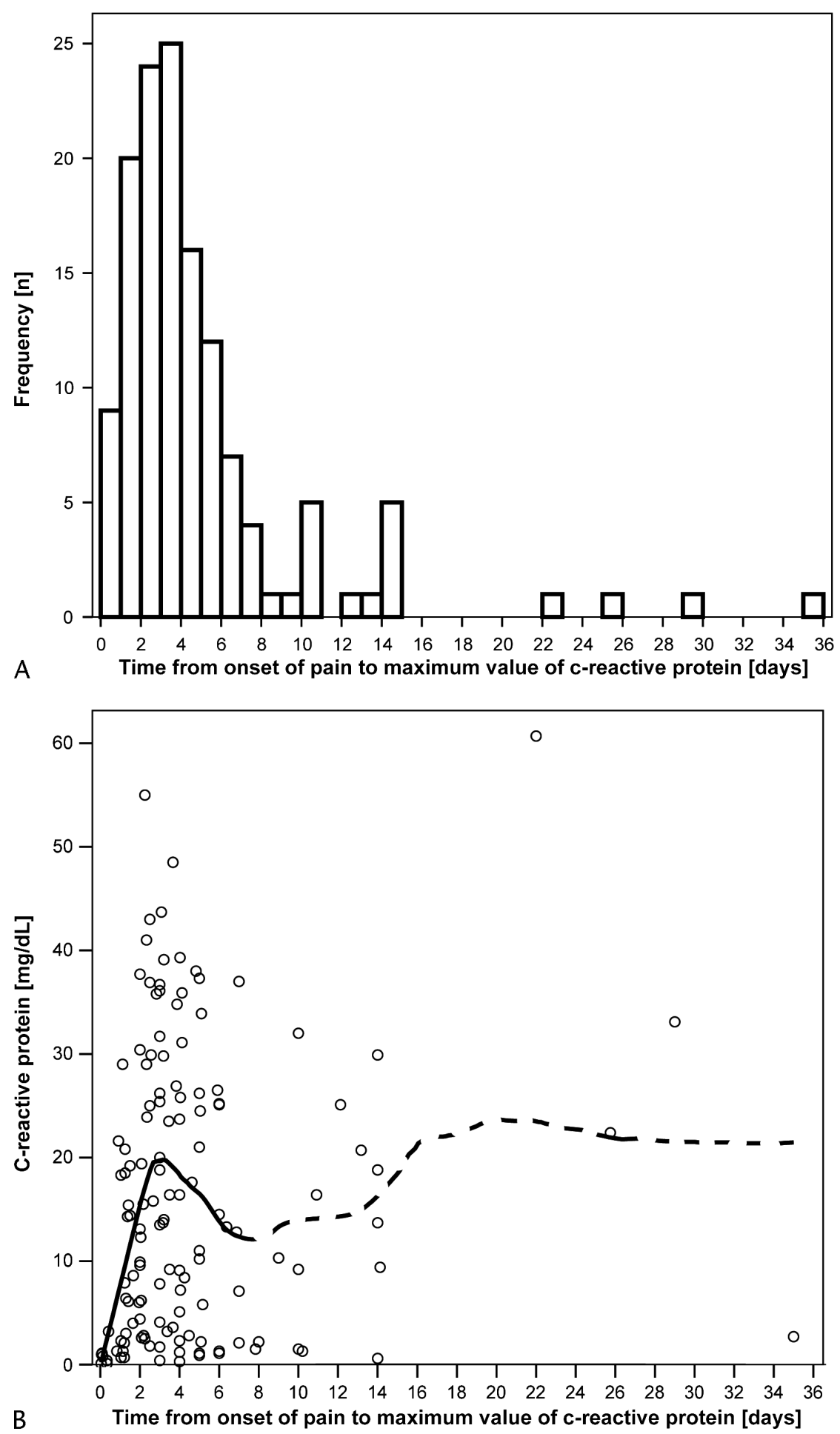

FIGURE 3. Bar plot showing the frequency of maximum levels of C-reactive protein since onset of pain (A). Scatter plot showing the absolute maximum levels of C-reactive protein since onset of pain (B). Solid and partly dotted lines depict the smoothed moving average.

There was an inappropriate discriminative performance of the single parameters CRP (c-index, 0.50) and lipase (c-index, 0.64 ) for the prediction of ICU admission. However, because relevant time dependencies were apparent in the descriptive analysis, an additional impact of the pain-to-admission time (c-index, 0.63) on the predictive performance of these markers was hypothesized. Therefore, a multiple logistic regression analysis including the admission values for CRP and serum lipase and the corresponding interactions to the pain-to-admission time was performed. This interaction model showed a substantial 

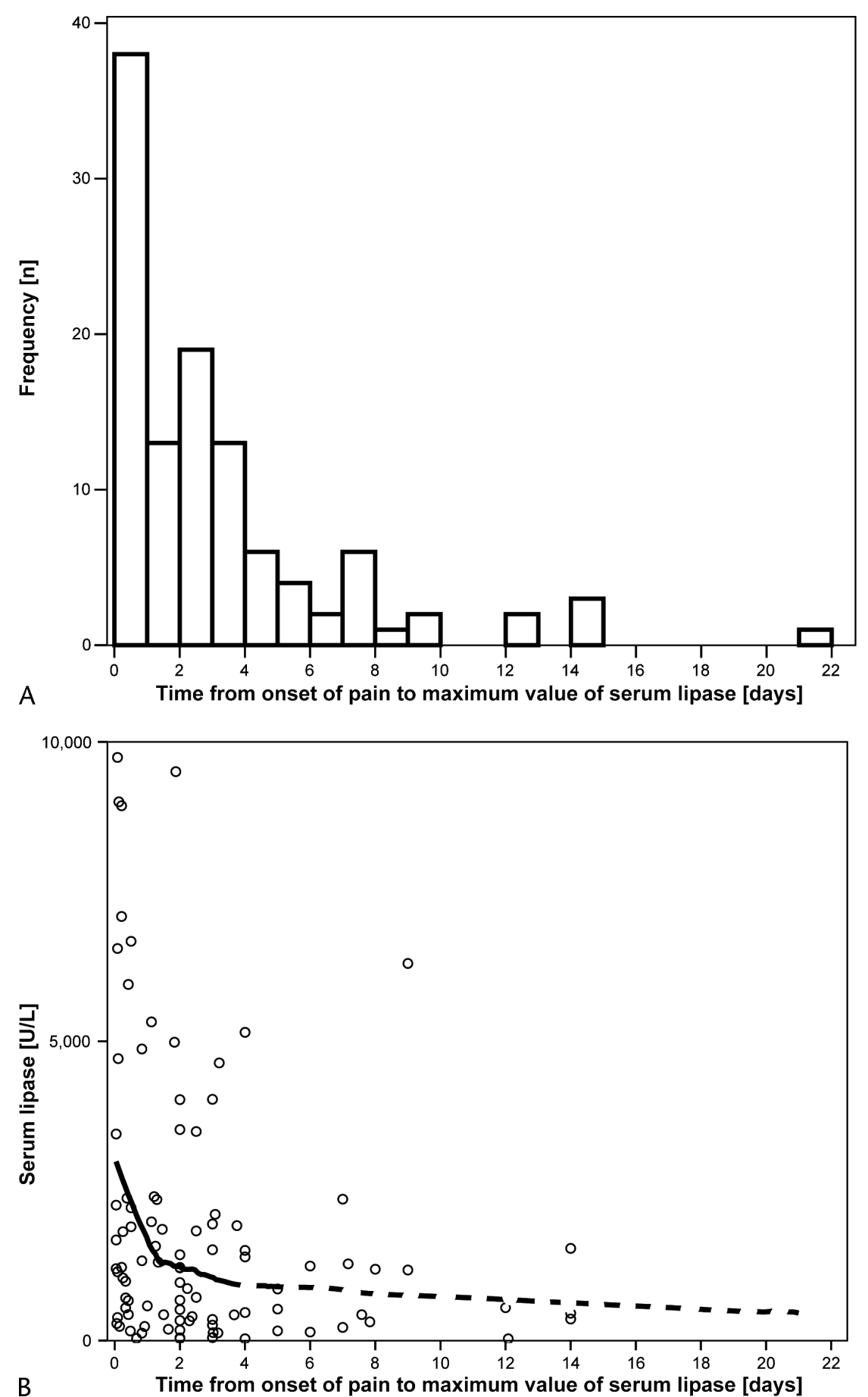

FIGURE 4. Bar plot showing the frequency of maximum levels of serum lipase since onset of pain (A). Scatter plot showing the absolute maximum levels of serum lipase since onset of pain (B). Solid and partly dotted lines depict the smoothed moving average.

discriminative ability regarding ICU admission (c-index, 0.706; $95 \%$ CI, 0.570-0.841; $P=0.006$; see Fig. 2, Supplemental Digital Content 2, http://links.lww.com/MPA/A204). With regard to practical applications, we developed a nomogram to predict need for ICU admission based on the parameters CRP, lipase, and pain-to-admission time (see Fig. 3, Supplemental Digital Content 2, http://links.lww.com/MPA/A205).

\section{Patients' Suspected Diagnosis and Self-Therapy}

The most frequent causes of pain suspected by the patients were diseases of the stomach/bowel (52/127, 41\%). Only 29/127 (23\%) of the patients associated their complaints to any affection of the pancreas. Of the 127 patients who answered this question, $9(7 \%)$ suspected an impairment of the liver, $21(17 \%)$ suspected various extraintestinal disorders, and $16(13 \%)$ patients had "no idea" at all. 
Of the 137 patients, $72(53 \%)$ did not start any therapy on their own, $52(38 \%)$ took some kind of drugs. Among these, $33(24 \%)$ used pain reliever, $12(9 \%)$ proton pump inhibitors, $8(6 \%)$ used butylscopolamine, and $8(6 \%)$ took other drugs (multiple answers possible); 13 (10\%) used nonpharmacological therapies including tea drinking and hot-water bag.

\section{Knowledge About the Pancreas and Pancreatitis}

Of the 130 patients who answered the questions about their knowledge on the pancreas and AP, $112(86 \%)$ were aware that they have a pancreas; of the 128 patients, $80(63 \%)$ knew the correct anatomic position and $69(54 \%)$ also knew the right physiological function of this organ. Regarding AP, 81/112 $(72 \%)$ of the patients who knew that they have a pancreas were aware of this disease and 45/81 (56\%) of those patients recognized that this is a potentially severe and fatal disease.

In univariate analysis, patients with a first-degree relative experiencing a pancreatic disease knew significantly more often that they have a pancreas compared to patients without pancreatic ill relatives [21/21 (100\%) vs 88/106 (83\%); $P=0.039]$. Patients with diabetes mellitus significantly less often knew that they have a pancreas than patients not experiencing diabetes mellitus [15/21 (71\%) vs 95/107 (89\%); $P=0.043]$. Etiology of AP, age, sex, residency, and recurrent attack of AP showed no substantial influence on the knowledge about the pancreas. $P$ values are taken from the adjusted multivariable logistic regression model. In multivariable analysis including these parameters, only diabetes mellitus had a statistical significant negative influence on the knowledge about the existence of the pancreas (odds ratio, $0.254 ; 95 \% \mathrm{CI}, 0.067-0.967 ; P=0.045$ ).

Regarding the knowledge of the entity of AP, multivariable logistic regression analysis including the parameters sex, age, place of residence, alcoholic etiology, biliary etiology, recurrent attack of AP, family history of pancreatic disease, and diabetes mellitus showed only alcoholic etiology (odds ratio, 3.670; 95\% CI, 1.236-10.900; $P=0.019$ ) as an independent factor to know that a disease "acute pancreatitis" exists.

\section{DISCUSSION}

This article provides data regarding pain-to-admission time and patients' knowledge on AP, taken from a prospective multicenter study by our group (PROST study). ${ }^{2}$

Early prediction of severity of AP is of high importance for triage which patient has to be treated as an outpatient, inpatient, or even ICU-patient. A lot of research has been done on this subject and a lot of single parameters, for example, CRP, procalcitonin, interleukin-6, D-dimer, and serum urea nitrogen (SUN) have been suggested as predictors for disease severity. ${ }^{10-14}$ Because single parameters are susceptible for failure and no single parameter provides appropriate predictive capabilities, many scores have been introduced, for example, Ranson score, Balthazar CT severity index, Pancreatitis Outcome Prediction (POP) score, bedside index for severity in AP (BISAP) score, and harmless AP score (HAPS) to predict a severe or harmless course of AP ${ }^{4,15-19}$ Furthermore, several mostly unknown scores, including artificial neural networks, have been developed. ${ }^{20-24}$ However, there are different shortcomings with all of them. Some parameters are not obtainable everywhere or are expensive, some scores are complex, have either a low negative predictive value (NPV) or low positive predictive value (PPV), or predict outcome not earlier than 48 hours after admission. Therefore, our study focused on early and widely available predictors including simple anamnestic data, which might be underrepresented in the current scores. According to our data, short pain-to-admission time correlates with higher probability of ICU treatment. Furthermore, short painto-admission time is associated with high pain levels, low values of CRP on admission, and high values of serum lipase on admission. Andersson et $\mathrm{al}^{24}$ recently presented an artificial neural network model for prediction of severity of AP, including for the first time the duration of pain as one of the predicting factors and showing shorter duration of pain until arrival at the emergency department for severe cases. The primary analysis of our data showed high serum lipase values on admission, among others, as a predictor for ICU treatment. ${ }^{2}$ Whereas CRP has shown to be a prognostic factor for severity of AP, the value of lipase frequently is considered to have diagnostic rather than predictive qualities. ${ }^{25-27} \mathrm{CRP}$ and serum lipase are dependent on the duration of AP and show contrary courses: Serum lipase increases 4 to 8 hours after onset of AP and reaches a maximum at approximately 24 hours, followed by a continuous decrease for 8 to 14 days. In contrast, CRP has a delayed increase with a predictive value starting at approximately 36 hours and a maximal value at 72 to 96 hours after onset of symptoms. ${ }^{10,25}$ According to these findings, serum lipase should have a high PPV and NPV within the first approximately 24 hours, followed by a high PPV but low NPV. In contrast, CRP has a low NPV within the first roughly 72 hours, followed by a high NPV and high PPV after approximately 96 hours. Therefore, a combination of CRP and lipase in dependency of the time since the onset of pain might provide a useful prediction formula. ${ }^{10,28-30}$ Our data suggest that serum lipase and CRP on admission in dependency of the time since onset of pain is a suitable predictor for the need of ICU treatment. This underlines that predictive capabilities of several markers markedly depend on the time since onset of symptoms as previously postulated. ${ }^{30}$

We choose ICU admission as end point because it is well defined and available for all patients, in contrast to the Atlanta severity classification or complex scores like APACHE-II, Ranson, or BISAP score. ${ }^{31,32}$ Furthermore, some patients might not have developed severe AP according to the Atlanta classification due to the close monitoring and intensive treatment in the ICU. Except for one patient, all patients who were transferred to the ICU had either severe AP according to the Atlanta classification $(17 / 40 ; 43 \%)$ or had at least 1 well-established prognostic factor regarding severe AP on admission. These prognostic factors included the following values: serum glucose greater than $250 \mathrm{mg} / \mathrm{dL}$ in patients without diabetes mellitus $(12 / 40 ; 30 \%)$, hematocrit greater than $44 \%(14 / 40 ; 35 \%)$, and SUN greater than $22 \mathrm{mg} / \mathrm{dL}$ on admission $(23 / 40 ; 58 \%)$ or a rise of SUN within 24 hours after admission $(2 / 40 ; 5 \%){ }^{15,33-35}$

The only exception mentioned previously was an 89-yearold lady experiencing biliary AP with cardiac events in her medical history. Therefore, all patients had a good indication to be transferred to the ICU.

In a region with unrestricted and fast access to medical service, pain-to-admission time might be mainly determined by patients' assessment of symptoms and severity of the causes supposed. Therefore, the questionnaire investigated patients' suspected etiology of complaints and their knowledge about the pancreas and AP. Several studies demonstrated that in patients with acute coronary syndrome or stroke educational campaigns resulting in better patients' awareness for early symptoms can shorten the time between appearance of first symptoms and hospital admission. ${ }^{36-38}$ Furthermore, shortened time between onset of symptoms and beginning treatment has been shown to improve outcome in these patients. ${ }^{39,40}$ Although cardiovascular diseases are much more frequent and have a higher mortality, earlier beginning of treatment in patients experiencing AP could also improve outcome in these patients. Similar to 
cardiovascular diseases, there is - despite a lack of supporting data - universal agreement that aggressive and early treatment (ie, fluid resuscitation) is critical in the therapy for AP. ${ }^{41} \mathrm{Be}-$ cause there are no data on knowledge and awareness of early symptoms in AP, we also investigated the knowledge about the existence, anatomy, and function of the pancreas as well as early symptoms of AP. Although $86 \%$ of patients with AP knew that they have a pancreas, only $72 \%$ of these patients knew that the pancreas can get inflamed and only $56 \%$ recognized that this is a potential fatal disease. This implicates that the knowledge on the pancreas and pancreatitis is poor.

The median pain-to-admission time was 27 hours. Because early fluid resuscitation is one of the most important parts of therapy for AP, an admission to the hospital and initiation of fluid administration as soon as possible is desirable. ${ }^{6-8}$ A study by Brown et $\mathrm{al}^{34}$ showed a hematocrit level greater than or equal to $44 \%$ on admission and failure of admission hematocrit to decrease at 24 hours after admission were the best predictors of necrotizing pancreatitis, also indicating the value of early therapy. Furthermore, the mostly used and most useful severity scores are calculated within the first 48 hours and patients with AP who already had an organ failure at first presentation had a significant higher mortality. ${ }^{42-44}$ Therefore, the painto-admission time should be shortened. Especially patients with increased risks to develop AP, for example, problem drinkers and patients with cholecystolithiasis should know about their pancreas and pancreatitis. Only patients admitted because of alcohol-induced AP, but not patients with biliary AP, knew more often that pancreatitis exists compared to patients with AP due to other reasons. This suggests that physicians fail to inform patients at the diagnosis of cholecystolithiasis about the risk of biliary AP. Assuming a prevalence of cholecystolithiasis of $20 \%$ and an incidence of biliary pancreatitis of 14.8/100,000/y in Germany, the risk of patients with cholecystolithiasis to develop an episode of biliary pancreatitis is $0.08 \%$ per year, that is, 1 of 1333 per year. ${ }^{2,45-47}$ We realize that this is a low number, however, considering rising life expectancy, the lifetime risk is much higher. Furthermore, biliary pancreatitis might be fatal for the individual patient and the medical education is very simple. Against our expectations, the knowledge on the pancreas in patients with diabetes mellitus was worse compared to patients without diabetes. This might be because patients experiencing diabetes focus on the endocrine function of the pancreas.

As the elimination of the causing agent is the only casual therapy for AP and a high percentage of AP is caused by immoderate alcohol intake, Takeyama ${ }^{48}$ observed the drinking habits of patients after the first attack of AP. They found a higher risk of recurrence, progression to chronic pancreatitis, and development of diabetes in patients who maintained their drinking level compared to patients who discontinued or reduced alcohol consumption. Also, another study supports these findings. ${ }^{49}$ In our study, $90.0 \%$ of patients with alcohol-induced AP previously had received admonitions to reduce immoderate alcohol consumption.

The efforts to improve patients' outcome are not only based on medical but also on economic reasons. Two recent US studies calculated the annual direct medical costs in the United States to $\$ 2.2$ billion for AP and $\$ 3.7$ billion for AP and chronic pancreatitis. ${ }^{50,51}$ Therefore, the awareness of the pancreas, of pancreatitis, and of the impact of alcohol consumption and smoking on the risk of acute and chronic pancreatitis must be raised in our population, especially in patients with risk factors also including cholecystolithiasis. ${ }^{52}$

Summing up, AP is a not infrequent, potential fatal and expensive disease. The first hours of the disease seem to be the most susceptible time for effective therapy. With a median pain-to-admission time of 27 hours, the first important day is lost by the patient himself. Educational efforts on patients' awareness could shorten the pain-to-admission time and therefore gain valuable time for the therapy for AP. Especially people on risk, for example, patients with cholecystolithiasis and/or immoderate alcohol consumption, should be educated regarding AP.

There are some limitations of this study. Despite the multicenter approach of the study, it was conducted in a limited area, the observation period was only 54 days, and only 188 patients were included. We do not know the exact reasons for ICU admissions of the individual patients but all except one had a severe AP according to the Atlanta classification or at least one prognostic factor regarding a severe course. However, conclusions regarding the prognostic capability of the admission values for serum lipase and CRP and the corresponding interactions to the pain-to-admission time have to be confirmed in larger and interventional studies and different populations.

We conclude that the knowledge about AP in hospitalized AP patients is poor with an inappropriately long painto-admission time. To improve the outcome of patients with AP by starting therapy as early as possible, the pain-toadmission time should be shortened and the knowledge about AP should be improved, especially in patients at risk. Serum lipase and CRP have appropriate predictive value regarding severity of AP only when used in the context of the time since onset of pain.

\section{ACKNOWLEDGMENT}

The authors thank the following individuals who made special contributions to the success of this project (alphabetically by institution):

Krankenhaus Agatharied: Dr A. Pohlmann and Dr A. Wahlländer. Argirov Klinik Starnberger See: Prof Dr D. J. Beuckelmann and Dr D. Ewald.

Asklepios Stadtklinik Bad Tölz: Dr F. Dorfmeister and Dr M. Volpert.

Krankenhaus Barmherzige Brüder München: Dr M. Anzinger, Dr G. Bischoff, and Dr E. Mathavan.

Klinikum Bogenhausen: Dr T. Leimbach and Prof Dr W. Schepp.

Klinikum Dachau: Dr G. Kachel and Dr Y. Turbanisch.

Klinik Dorfen: Dr L. Rudolf.

Klinikum Dritter Orden: PD Dr A. Eigler and Dr C. Kaiser.

Kreisklinik Ebersberg: Dr G. Feder and Dr W. Kühner.

Kreiskrankenhaus Erding: Prof Dr H.P. Emslander and

Dr M. Koch.

Klinikum Freising: Prof Dr E. Schulte-Frohlinde,

Dr S. Ringmaier, and Prof Dr J. Phillip

Klinikum Fürstenfeldbruck: Prof Dr R. Eissele and Dr B. Jacob.

Klinikum der Universität München Campus Großhadern:

Prof Dr B. Göke and Prof Dr A. Wagner.

Klinikum Harlaching: Dr G. Berna.

Klinikum der Universität München Campus Innenstadt: PD Dr B. Otto.

Internistische Klinik Dr Müller: Prof Dr Dr M. Gross.

Klinikum Neuperlach: Dr M. Blöchinger and Dr K. Wenke.

Klinikum München Pasing: Dr D. Bergemann and

Dr F. Neumann

Klinik München Perlach: Dr M. Bergholtz and Dr F. Martin.

Klinikum rechts der Isar der Technischen Universität München. Sharon Page.

Klinikum Rosenheim: Dr J. Boedecker and Dr H. Huff.

Rotkreuzklinikum München: Dr E. Ahrens, Prof Dr P. Born, and Dr S. Petersen.

Privatklinik Dr Robert Schindlbeck: Dr M. Brennenstuhl and Dr U. Middeler. 


\author{
Klinikum Schwabing: Dr H. J. Fischer, Dr E. Höcherl, and \\ Dr I. Knetsch. \\ Klinikum Starnberg: PD Dr A. Klauser and \\ Prof Dr P. Trenkwalder. \\ Kreisklinik Wolfratshausen: $\mathrm{Dr}$ W. Hiller and T. Leis.
}

\section{REFERENCES}

1. Lankisch PG, Assmus C, Maisonneuve P, et al. Epidemiology of pancreatic diseases in Luneburg County. A study in a defined german population. Pancreatology. 2002;2:469-477.

2. Phillip V, Huber W, Hagemes F, et al. Incidence of acute pancreatitis does not increase during Oktoberfest, but is higher than previously described in Germany. Clin Gastroenterol Hepatol. 2011;9:995-1000.

3. Whitcomb DC. Clinical practice. Acute pancreatitis. $N$ Engl J Med. 2006;354:2142-2150

4. Harrison DA, D'Amico G, Singer M. The Pancreatitis Outcome Prediction (POP) score: a new prognostic index for patients with severe acute pancreatitis. Crit Care Med. 2007;35:1703-1708.

5. Banks PA, Freeman ML. Practice guidelines in acute pancreatitis. Am J Gastroenterol. 2006;101:2379-2400.

6. Warndorf MG, Kurtzman JT, Bartel MJ, et al. Early fluid resuscitation reduces morbidity among patients with acute pancreatitis. Clin Gastroenterol Hepatol. 2011;9:705-709.

7. Otsuki M, Hirota M, Arata S, et al. Consensus of primary care in acute pancreatitis in Japan. World J Gastroenterol. 2006;12:3314-3323.

8. Gardner TB, Vege SS, Chari ST, et al. Faster rate of initial fluid resuscitation in severe acute pancreatitis diminishes in-hospital mortality. Pancreatology. 2009;9:770-776.

9. Takeda K, Takada T, Kawarada Y, et al. JPN Guidelines for the management of acute pancreatitis: medical management of acute pancreatitis. J Hepatobiliary Pancreat Surg. 2006;13:42-47.

10. Schutte K, Malfertheiner P. Markers for predicting severity and progression of acute pancreatitis. Best Pract Res Clin Gastroenterol. 2008;22:75-90.

11. Woo SM, Noh MH, Kim BG, et al. Comparison of serum procalcitonin with Ranson, APACHE-II, Glasgow and Balthazar CT Severity Index Scores in predicting severity of acute pancreatitis. Korean J Gastroenterol. 2011;58:31-37.

12. Pezzilli R, Billi P, Miniero R, et al. Serum interleukin-6, interleukin-8, and beta 2-microglobulin in early assessment of severity of acute pancreatitis. Comparison with serum C-reactive protein. Dig Dis Sci. 1995;40:2341-2348.

13. Ke L, Ni HB, Tong ZH, et al. D-dimer as a marker of severity in patients with severe acute pancreatitis. J Hepatobiliary Pancreat Sci. 2012;19:259-265.

14. Wu BU, Johannes RS, Sun X, et al. Early changes in blood urea nitrogen predict mortality in acute pancreatitis. Gastroenterology. 2009; 137:129-135.

15. Ranson JH, Rifkind KM, Roses DF, et al. Objective early identification of severe acute pancreatitis. Am J Gastroenterol. 1974;61:443-451.

16. Balthazar EJ, Ranson JH, Naidich DP, et al. Acute pancreatitis: prognostic value of CT. Radiology. 1985;156:767-772.

17. Wu BU, Johannes RS, Sun X, et al. The early prediction of mortality in acute pancreatitis: a large population-based study. Gut. 2008;57:1698-1703.

18. Lankisch PG, Weber-Dany B, Hebel K, et al. The harmless acute pancreatitis score: a clinical algorithm for rapid initial stratification of nonsevere disease. Clin Gastroenterol Hepatol. 2009;7:702-705; quiz 607.

19. Pavlidis TE, Pavlidis ET, Sakantamis AK. Advances in prognostic factors in acute pancreatitis: a mini-review. Hepatobiliary Pancreat Dis Int. 2010;9:482-486.

20. Mentula P, Kylanpaa ML, Kemppainen E, et al. Early prediction of organ failure by combined markers in patients with acute pancreatitis. Br J Surg. 2005;92:68-75
21. Ueda T, Takeyama Y, Yasuda T, et al. Simple scoring system for the prediction of the prognosis of severe acute pancreatitis. Surgery. 2007;141:51-58

22. King NK, Powell JJ, Redhead D, et al. A simplified method for computed tomographic estimation of prognosis in acute pancreatitis. Scand J Gastroenterol. 2003;38:433-436.

23. Singh VK, Wu BU, Bollen TL, et al. A prospective evaluation of the bedside index for severity in acute pancreatitis score in assessing mortality and intermediate markers of severity in acute pancreatitis. Am J Gastroenterol. 2009;104:966-971.

24. Andersson B, Andersson R, Ohlsson M, et al. Prediction of severe acute pancreatitis at admission to hospital using artificial neural networks. Pancreatology. 2011;11:328-335.

25. Pongprasobchai S, Jianjaroonwong V, Charatcharoenwitthaya $\mathrm{P}$, et al Erythrocyte sedimentation rate and C-reactive protein for the prediction of severity of acute pancreatitis. Pancreas. 2010;39:1226-1230.

26. Cornett DD, Spier BJ, Eggert AA, et al. The causes and outcome of acute pancreatitis associated with serum lipase $>10,000 \mathrm{U} / \mathrm{L}$. Dig Dis Sci. 2011;56(11):3376-3381

27. Huber W, Schmid RM. [Diagnosis and treatment of acute pancreatitis: current recommendations]. Internist (Berl). 2011;52:823-832.

28. Tietz NW, Shuey DF. Lipase in serum - the elusive enzyme: an overview. Clin Chem. 1993;39:746-756.

29. Lankisch PG, Buschmann-Kaspari H, Otto J, et al. Correlation of pancreatic enzyme levels with the patient's recovery from acute edematous pancreatitis. Klin Wochenschr. 1990;68:565-569.

30. Huber W, Umgelter A, Schuster T, et al. The Pancreatitis Outcome Prediction (POP) score: how about hematocrit and leukopenia? Crit Care Med. 2007;35:2670-2671; author reply 2671-2672.

31. Bradley EL 3rd. A clinically based classification system for acute pancreatitis. Summary of the International Symposium on Acute Pancreatitis, Atlanta, Ga, September 11 through 13, 1992. Arch Surg. 1993;128:586-590.

32. Di Fabio F, Abu Hilal M, Johnson CD. Acute pancreatitis: mild, severe or potentially fatal. Pancreatology. 2011;11:373-375.

33. Lankisch PG, Blum T, Bruns A, et al. Has blood glucose leve measured on admission to hospital in a patient with acute pancreatitis any prognostic value? Pancreatology. 2001;1:224-229.

34. Brown A, Orav J, Banks PA. Hemoconcentration is an early marker for organ failure and necrotizing pancreatitis. Pancreas. 2000;20:367-372.

35. Wu BU, Bakker OJ, Papachristou GI, et al. Blood urea nitrogen in the early assessment of acute pancreatitis: an international validation study. Arch Intern Med. 2011;171:669-676.

36. Naegeli B, Radovanovic D, Rickli H, et al. Impact of a nationwide public campaign on delays and outcome in Swiss patients with acute coronary syndrome. Eur J Cardiovasc Prev Rehabil. 2011;18:297-304

37. Fogle CC, Oser CS, McNamara MJ, et al. Impact of media on community awareness of stroke warning signs: a comparison study. J Stroke Cerebrovasc Dis. 2010;19:370-375.

38. Mullen PD, Mains DA, Velez R. A meta-analysis of controlled trials of cardiac patient education. Patient Educ Couns. 1992;19:143-162.

39. Berger PB, Ellis SG, Holmes DR Jr, et al. Relationship between delay in performing direct coronary angioplasty and early clinical outcome in patients with acute myocardial infarction: results from the global use of strategies to open occluded arteries in Acute Coronary Syndromes (GUSTO-IIb) trial. Circulation. 1999;100:14-20.

40. Marler JR, Tilley BC, Lu M, et al. Early stroke treatment associated with better outcome: the NINDS rt-PA stroke study. Neurology. 2000;55:1649-1655.

41. Gardner TB, Vege SS, Pearson RK, et al. Fluid resuscitation in acute pancreatitis. Clin Gastroenterol Hepatol. 2008;6:1070-1076.

42. Hirota M, Takada T, Kawarada Y, et al. JPN Guidelines for the management of acute pancreatitis: severity assessment of acute pancreatitis. J Hepatobiliary Pancreat Surg. 2006;13:33-41. 
43. Chatzicostas C, Roussomoustakaki M, Vlachonikolis IG, et al. Comparison of Ranson, APACHE II and APACHE III scoring systems in acute pancreatitis. Pancreas. 2002;25:331-335.

44. Isenmann R, Rau B, Beger HG. Early severe acute pancreatitis: characteristics of a new subgroup. Pancreas. 2001;22:274-278.

45. Kratzer W, Mason RA, Kachele V. Prevalence of gallstones in sonographic surveys worldwide. J Clin Ultrasound. 1999;27:1-7.

46. Lammert F, Sauerbruch T. Mechanisms of disease: the genetic epidemiology of gallbladder stones. Nat Clin Pract Gastroenterol Hepatol. 2005;2:423-433.

47. Volzke H, Baumeister SE, Alte D, et al. Independent risk factors for gallstone formation in a region with high cholelithiasis prevalence. Digestion. 2005;71:97-105.
48. Takeyama Y. Long-term prognosis of acute pancreatitis in Japan. Clin Gastroenterol Hepatol. 2009;7:S15-S17.

49. Nordback I, Pelli H, Lappalainen-Lehto R, et al. The recurrence of acute alcohol-associated pancreatitis can be reduced: a randomized controlled trial. Gastroenterology. 2009;136:848-855.

50. Everhart JE, Ruhl CE. Burden of digestive diseases in the United States Part III: Liver, biliary tract, and pancreas. Gastroenterology. 2009;136:1134-1144.

51. Fagenholz PJ, Fernandez-del Castillo C, Harris NS, et al. Direct medical costs of acute pancreatitis hospitalizations in the United States. Pancreas. 2007;35:302-307.

52. Yadav D. Recent advances in the epidemiology of alcoholic pancreatitis. Curr Gastroenterol Rep. 2011;13:157-165. 